\title{
Efficient cooperative compressive spectrum sensing by identifying multi-candidate and exploiting deterministic matrix
}

\author{
Jia Li ${ }^{1}$, Qiang Wang ${ }^{1 *}$, Wenjie Yan ${ }^{2}$ and Yi Shen ${ }^{1}$
}

\begin{abstract}
Cooperative spectrum sensing exploits the spatial diversity to improve the detection of occupied channels in cognitive radio networks (CRNs). Cooperative compressive spectrum sensing (CCSS) utilizing the sparsity of channel occupancy further improves the efficiency by reducing the number of reports without degrading detection performance. In this paper, we firstly and mainly propose the referred multi-candidate orthogonal matrix matching pursuit (MOMMP) algorithms to efficiently and effectively detect occupied channels at fusion center (FC), where multi-candidate identification and orthogonal projection are utilized to respectively reduce the number of required iterations and improve the probability of exact identification. Secondly, two common but different approaches based on threshold and Gaussian distribution are introduced to realize the multi-candidate identification. Moreover, to improve the detection accuracy and energy efficiency, we propose the matrix construction based on shrinkage and gradient descent (MCSGD) algorithm to provide a deterministic filter coefficient matrix of low $t$-average coherence. Finally, several numerical simulations validate that our proposals provide satisfactory performance with higher probability of detection, lower probability of false alarm and less detection time.
\end{abstract}

Keywords: CCSS; MOMMP; Multi-candidate identification; MCSGD; Deterministic matrix

\section{Introduction}

Cognitive radio (CR), as a promising technology to improve the spectrum efficiency in wireless communication, has drawn great attentions in recent years [1]. By detecting the channel states or spectrum holes, the CR users are allowed to communicate over the spectrum allocated to primary users (PUs) when they are not fully utilizing it. Spectrum sensing is the key technology for the above application [2]. Considering that the detection of a single CR user may suffer from the wireless channel fading and the hidden terminal problem, cooperative spectrum sensing (CSS) exploiting several spatially distributed CR users is introduced to improve the detection performance for whole CR networks (CRNs) [3].

Although CSS benefits from cooperative gain to improve the detection accuracy, cooperation overhead

\footnotetext{
*Correspondence: wangqiang@hit.edu.cn

1 Department of Control Science and Engineering, Harbin Institute of

Technology, 150001 Harbin, China

Full list of author information is available at the end of the article
}

is also incurred at the same time, which refers to any extra sensing time, delay, energy, reports, and operations devoted to CSS [4-6]. Traditionally, in centralized CSS, fusion center (FC) collects all sensing data and then makes decisions on channel occupancy. However, significant bandwidth is required due to the reporting over a wired high-speed backbone. One natural idea to address such issue is that each CR user reports a summary statistic. Considering that sparse signals can be exactly recovered from their fewer measurements than what are usually considered necessary in compressive sensing (CS) theory [7], cooperative compressive spectrum sensing (CCSS) using linear combinations as summary statistics is feasible. This is guaranteed by the recent studies on the actual spectrum utilization efficiency, which have revealed that a large portion of the licensed spectrum experiences low utilization, i.e., the channel occupancy is sparse. Thus, the energy efficiency of CRNs can be improved by reducing the number of reports.

\section{量 Springer}

(c) $2015 \mathrm{Li}$ et al. Open Access This article is distributed under the terms of the Creative Commons Attribution 4.0 International License (http://creativecommons.org/licenses/by/4.0/), which permits unrestricted use, distribution, and reproduction in any medium, provided you give appropriate credit to the original author(s) and the source, provide a link to the Creative Commons license, and indicate if changes were made. 
A novel CCSS model exploiting a frequency selective filter equipped at each CR user to linearly combine multiple channel states was firstly introduced in [8]. Meanwhile, two detection algorithms respectively based on matrix completion and jointly sparse recovery were also proposed to recover the channel occupancy, while the relatively faster jointly sparse recovery algorithm still involves multiple constrained optimization sub-problems. Actually, many researchers have paid their attention to the design of jointly sparse recovery algorithm. In [9], a decentralized consensus optimization algorithm was derived to estimate the spectrum, where an $l_{1}$-regularized least square problem was solved at each CR user. To further save the transmission burden by exchanging only the estimated joint support with neighbor users, Ling et al. [10] introduced several centralized or decentralized reweighed $l_{q}$ algorithms $(q=1,2)$. However, the above algorithms are of too high computational complexity, which limits their application in real-time and large-scale system. In [11], for the support with infinite cardinality, a continuous to finite (CTF) block consisting construction of a finite frame and recovering the joint support was proposed in recovery stage. However, in this paper, the support has finite cardinality, which makes the above block inapplicable. Recently, based on the idea of matching pursuit (MP) algorithm [12], three more efficient algorithms were consecutively proposed in [13, 14]. Unfortunately, they suffer from the non-orthogonality between selected atoms and residual data, which reduces the possibility of exactly detecting more occupied channels. Actually, just like the extension from MP to orthogonal MP(OMP) algorithm [15], the orthogonal projection (or least square) technique can overcome the above shortcoming. To further improve the efficiency, one simple and intuitive idea is to reduce the number of required iterations by identifying multicandidate in each iteration, resulting the multi-candidate orthogonal matrix matching pursuit (MOMMP) algorithm. One such algorithm, based on the subspace pursuit (SP) algorithm [16], has been introduced in [17]. However, it requires a priori knowledge of the unknown and varying sparsity, which make it practically inapplicable. Respectively motivated by the idea of generalized OMP (gOMP) [18] algorithm and that of stagewise OMP (StOMP) algorithm [19] and adaptive OMP (AOMP) algorithm [14], this paper proposes two simple approaches to realize the multi-candidate identification.

In addition to the design of detection algorithm, the selection of filter coefficient matrix also greatly affects the detection performance due to the essential jointly sparse recovery problem. According to the CS theory, one can guarantee an exact recovery when the filter coefficient matrix satisfies the restricted isometry property (RIP) [20]. Although random matrices under Gaussian distribution have been proven to obey the RIP with overwhelming probability, it is NP-hard to certify that a random matrix indeed satisfies RIP. Therefore, it is of interest to find or construct a deterministic RIP matrix. In addition, a deterministic matrix benefits the energy efficiency of CSS since its elements do not need to be transmitted and it reduces the number of required reports with detection accuracy constraint. Till now, some researchers have focused on the matrix construction [21, 22]. Along this research line, we propose a novel shrinkage function and gradient descent method to construct a deterministic matrix, which is of low $t$-coherence and improves the detection effectiveness and efficiency.

Under the assumption that the received wide-sense stationary signal is sparse in frequency domain, many researches focus on the reduction of sampling rate and the recovery of original signal $[23,24]$. Recently, power spectrum sensing drew much attention since the above sparsity assumption can be removed and an efficient recovery can be achieved at the same time. Along this research line, several sampling systems are introduced in [11, 25-31]. The multi-coset sampling used in [25-28] is based on the multi-rate signal processing and it employs parallel cosets that uniformly sample the signal at a decimated rate. Similarly, multiple branches are considered in $[11,30,32]$, where each branch employs a different periodic mixing function followed by low-pass filtering and low-rate uniform sampling or an integrate-and-dump process. In [29], the coprime sampling scheme is employed that involves using two sampling branches with sampling rates coprime with each other. And, a distributed scheme to estimate the power spectrum from lossy observations is proposed in [31]. Different from the above literatures, this paper considers the sparsity of channel occupancy, which presents the states of all channels. Instead of time samples, each CR users transmits fewer linear combinations of multiple channel states to the FC. Compared with $L$ compressions in each CR sensor [28], where $L$ is the number of samplers in each coset, this paper implements only one compression in each CR user and it can be described as a product of the filter coefficient matrix and the sparse channel occupancy. As for the detection step, similar to the works in [27,33], our proposal does not concentrate on the exact recovery of original signal either. Based on the energy distribution of row vectors of identification matrix, only the locations of nonzero rows are identified to obtain the sparse channel occupancy. Meanwhile, though the MOMMP algorithms iteratively execute one orthogonal projection step, the employment of multi-candidate identification has significantly saved the computational burden by decreasing the number of required iterations. In terms of detection from lossy measurements due to channel fading [31, 33], this paper performs extensive numerical experiments to validate the superiority of our proposal. 
The main contributions of this paper can be stated as:

1.) We propose the MOMMP algorithms to reduce detection time without degrading detection accuracy, in which multi-candidate identification and orthogonal projection are respectively implemented in each iteration to detect several occupied channels and recover partial state data;

2.) We introduce two simple approaches to realize the multi-candidate identification. In threshold-based MOMMP (TMOMMP) algorithm, a certain number of coordinates are identified in each iteration, and a threshold-based scheme is then utilized to redeclare the occupied channels. Furthermore, in MOMMP based on Gaussian distribution (MOMMP-GD) algorithm, an uncertain number of coordinates are identified in each iteration according to the distribution of energy of row vectors in identification matrix;

3.) We propose the matrix construction based on shrinkage and gradient descent (MCSGD) algorithm to construct a deterministic filter coefficient matrix, which is of low $t$-averaged coherence;

4.) The advantages of integrating MOMMP and MCSGD algorithms are higher probability of exactly detecting occupied channels, less detection time, and unnecessary transmission of the components of deterministic filter coefficient matrix.

The rest of this paper is organized as follows. The second section briefly reviews the CS theory and the system model of CCSS. In the third section, the proposed TMOMMP and MOMMP-GD algorithms are described in detail. The proposed MCSGD algorithm providing deterministic matrix as well as its experimental validations are presented in the fourth section. Several numerical experiments validating detection accuracy and time are shown in the fifth section, followed by the Conclusions section.

\section{System model of cooperative compressive spectrum sensing (CCSS) Compressive sensing (CS) theory}

Compressive sensing, as an emerging framework for efficient sampling and compression of sparse signals, demonstrates that sparse signals can be recovered from their fewer measurements than what are considered necessary [7]. $x \in R^{n}$ is said to be a sparse signal with sparsity $s(s \ll$ $n)$ if $|\Gamma| \leq s$, where $\Gamma=\left\{i \mid x_{i} \neq 0, i=1, \ldots, n\right\}$ denotes its support. Assume the measurement signal $y \in R^{p}$ is perturbed as

$$
y=\Phi x+e
$$

where $\Phi \in R^{p \times n}(p<n)$ is the measurement matrix with each normalized column termed as atom, and $e$ is a noise vector. The recovery of $x$ can be achieved by solving the following $l_{0}$ (or pseudo)-norm minimization problem

$$
\tilde{x}=\arg \min _{x}\|x\|_{0} \text { s.t. }\|y-\Phi x\|_{2} \leq \varepsilon
$$

\section{System model}

We consider a CRN with $m$ CR users that locally sense the state of $n$ channels. A channel is either occupied by its licensed PU or unoccupied, corresponding to the states 1 and 0 , respectively. Traditionally, each CR user orderly sends $n$ states to the centralized FC to make global decisions on channel occupancy. Suppose that there is at most one PU on each channel and only $s(s<n)$ channels are occupied, the channel occupancy can be described as a vector of length $n$ and sparsity $s$. Due to each CR user can only sense limited channels at a time and the limitation of energy reservation and hardware implementation, it is impossible for each CR user to detect all $n$ channels simultaneously.

The CCSS model in [8] proposes to exploit a frequency selective filter in each CR user to obtain $p(p<n)$ linear combination of multiple channel states. In this way, each CR user will send only $p$ linear combinations to FC, which can be described as a compression by a filter coefficient matrix $F \in R^{p \times n}$. We set a $n \times n$ diagonal matrix $R$ to represent the states of $n$ channels, where the $j(1 \leq j \leq n)$ th diagonal entry is the state of the $j$ th channel ( 0 or 1$)$. The $p$ reports from each CR user forms the vector $F \cdot \operatorname{diag}(R) \in$ $R^{p \times 1}$, whose elements are orderly collected by the FC. The channel gain between $m$ CR users and $n$ channels can be described in an $m \times n$ channel gain matrix $C$ [34], whose $(i, j)$ th component is written as

$$
C_{i, j}=P_{j}\left(d_{i, j}\right)^{-\alpha / 2}\left|h_{i, j}\right|
$$

where $P_{j}$ is the transmitted power of PU using $j$ th channel, $d_{i, j}$ denotes the distance between the PU using $j$ th channel and $i$ th CR user, $\alpha$ is the propagation loss factor, and $h_{i, j}$ is the channel fading gain. Here, we consider $h_{i, j}=1, \forall i, j$ for additive white Gaussian noise (AWGN) channel and $\left|h_{i, j}\right|$ following independent Rayleigh distribution for Rayleigh channel [34].

Utilizing a filter coefficient matrix $F \in R^{p \times n}$ on each CR user, we obtain the measurement data $M \in R^{p \times m}$. Considering the inevitable perturbation in transmission, $M$ is further perturbed as

$$
M=F R C^{T}+N=F X+N
$$

where $X=R C^{T} \in R^{n \times m}$ denotes the state data, and $N \in R^{p \times m}$ is a Gaussian noise in reporting channel. In this way, the number of reports is reduced from $n m$ to $p m$, which saves the energy consuming in sharing channel information. Generally, the transmission failure will possi- 
bly make some entries of $M$ missing. Therefore, the entries of received data $M^{E}$ are described as

$$
M_{i, j}^{E}=\left\{\begin{array}{cc}
M_{i, j}, & (i, j) \in E \\
0, & \text { otherwise }
\end{array}\right.
$$

where the index set $E$ denotes the successfully received locations in $M$.

According to the definition of $R$ and the expression in (4), the detection can be achieved by solving a jointly sparse recovery problem. To guarantee the uniqueness and existence of its solution, $F$ should satisfy the RIP [20]. Considering that CCSS only focuses on the locations of occupied channels, i.e., the nonzero elements in $\operatorname{diag}(R)$, we propose to alternatively find the nonzero rows of $X$.

\section{Multi-candidate orthogonal matrix matching pursuit (MOMMP) algorithms \\ The description of MOMMP algorithm}

Through an ideal transmission, i.e., lossless and noise-free, the received $M^{E}$ is written as

$$
M^{E}=M=F_{:, 1} X_{1,:}+\cdots+F_{:, n} X_{n,:}=\sum_{j \in \Gamma} F_{:, j} X_{j::}
$$

where $\Gamma \subset\{1, \cdot, n\}$ is the support of $\operatorname{diag}(R)$. The following identification matrix is firstly generated

$$
\begin{aligned}
B & =F^{T} M^{E}=F^{T} F X \\
& =\left[\begin{array}{c}
F_{:, 1}^{T} \\
\vdots \\
F_{:, n}^{T}
\end{array}\right]\left[\begin{array}{lll}
F_{:, 1} & \cdots & F_{:, n}
\end{array}\right] X
\end{aligned}
$$

According to the normalized columns in $F$, we further express the $i$ th row of $B$ as

$$
B_{i,:}=X_{i,:}+\sum_{j \neq i} F_{:, i}^{T} F_{:, j} X_{j,:}
$$

Thus, we achieve the perfect detection of occupied channels by locating all the nonzero rows of $B$ when $F$ is orthogonal. However, it is the fact $p<<$ that makes the above case impossible. To be specific, the lower bound of the absolute inner product of distinct columns in $F \in$ $R^{p \times n}$, i.e., the following Welch bound [35], makes all the rows of $B$ nonzero.

$$
\mu_{w}=\sqrt{(n-p)(n-1)^{-1} p^{-1}}
$$

Fortunately, the normalized columns of $F$ still guarantee that $B$ is a proximity to $X$ due to $\left|F_{:, i}^{T} F_{: j}\right|<<\left|F_{:, i}^{T} F_{:, i}\right|=1$ for all $i \neq j$. This means $i$ th row of $B$ is of large norm if $i$ th row of $X$ is nonzero. Therefore, we alternatively compute $\left\|B_{i,:}\right\|_{1}, 1 \leq i \leq n$ to judge whether $X_{i, \text { : }}$ is a null vector or not in sense of probability.

Following the idea of MMP and revised MMP (RMMP) algorithms in [13], we can detect one occupied channel in each iteration and terminate the detection after sth iteration. However, the actual sparsity $s$ is often unknown since it corresponds to the instantaneous spectrum occupancy of PUs which is dynamically varying. Although the typically available upper bound $s_{\max }$, which can be measured from the maximum spectrum utilization observed statistically over a time period, provides a conservative determination of $s$, the cost is still high due to $O(s)$ iterations in detection. In CRNs, the detection time shall be small enough compared with the channel coherence time and user dynamics, such that transmission opportunities can be utilized before the spectrum occupancy profile has changed. Thus, it is still worth investigating how to reduce the detection time without obviously degraded accuracy.

An intuitive idea is to reduce the number of required iterations by detecting multi-candidate occupied channels or identifying multi-candidate coordinates in each iteration, which has been investigated in [14]. Begin with the set of coordinates indexing detected channels $\Lambda=\emptyset$, we detect several occupied channels in the $l$ th iteration by identifying set $\Upsilon^{l}$, where $\left|\Upsilon^{l}\right| \geq 1$. This is followed by the set expansion $\Lambda=\Lambda \cup \Upsilon^{l}$. To combat the nonorthogonality between the selected atoms and the residual data [14], which conversely affects the possibility of exactly detecting more occupied channels, we implement an orthogonal projection process to compute the partial state data as $X_{\Lambda,:}=F_{:, \Lambda}^{\dagger} M$. Finally, the residual data is updated as $Y_{r}=M-F_{;, \Lambda} X_{\Lambda,:}$. In this way, the issue of repeated detection is addressed due to the following result

$$
\begin{aligned}
F_{:, \Lambda}^{T} Y_{r} & =F_{:, \Lambda}^{T}\left(M-F_{:, \Lambda} F_{:, \Lambda}^{\dagger} M\right) \\
& =F_{:, \Lambda}^{T}\left(F_{:, \Gamma} X_{\Gamma,:}-F_{:, \Lambda} F_{:, \Lambda}^{\dagger} F_{:, \Gamma} X_{\Gamma,:}\right) \\
& =F_{:, \Lambda}^{T}\left(F_{:, \Gamma-\Lambda} X_{\Gamma-\Lambda,:}-F_{:, \Lambda} F_{:, \Lambda}^{\dagger} F_{:, \Gamma-\Lambda} X_{\Gamma-\Lambda,:}\right)=0
\end{aligned}
$$

With the aid of multi-candidate identification and orthogonal projection techniques, we respectively improve the detection efficiency and effectiveness. In this paper, the algorithms following the above idea are collectively referred to as multi-candidates orthogonal MMP (MOMMP). In the following content, based on two different approaches achieving multi-candidate, we propose two simple MOMMP algorithms.

\section{Threshold-based MOMMP (TMOMMP) algorithm}

Inspired by the idea of the gOMP algorithm, we propose to detect $k$ occupied channels in each iteration, where $k \geq 1$ is a fixed integer. Based on the available $s_{\max }$, we terminate the detection by determining a maximum iteration number iter max $_{\text {. }}$

In each iteration, $\left.h^{l}=\left[\left\|B_{1,:}^{l}\right\|_{1}, \cdot,\left\|B_{n,:}^{l}\right\|_{1}\right]\right\}$ is firstly computed from the generated identification matrix $B^{l}=$ $F^{T} Y_{r}$. And then, $\Upsilon^{l}$ is identified as the set of coordinates corresponding to the $k$ largest entries of $h^{l}$ and $\Lambda$ is 
expanded as $\Lambda=\Lambda \cup \Upsilon^{l}$. Finally, the partial state data $X_{\Lambda, \text { : }}$ is computed and the residual data $Y_{r}$ is updated. In terms of its termination, we choose to preset the maximum iteration number iter $r_{\max }=\left\lceil s_{\max } / k\right\rceil$, where $\lceil a\rceil$ is the smallest integer that is larger than $a$.

According to expression in (10), no channel is repeatedly detected. Therefore, at least $s_{\max }$ channels are finally declared as occupied, demonstrating $\Gamma \subset \Lambda$. Different from the classic sparse signal recovery based on Lemma 3.1 in [19], CCSS aims to exactly obtain $\Gamma=\Lambda$ since that $\Lambda-\Gamma \neq \varnothing$ indicates the existence of false alarm. Thus, to reduce the probability of false alarm, it is necessary to refine the obtained $\Lambda$ or eliminate the coordinates in $\Lambda-$ $\Gamma$. In this paper, we propose the following threshold-based method to achieve the refinement

$$
\Lambda=\left\{i \mid\left\|X_{i,}\right\|_{1} \geq \lambda_{1}, i=1, \cdot, n\right\}
$$

where $\lambda_{1}>0$ is a preset decision threshold. Empirically, the threshold is set as $\lambda_{1}=\frac{\rho_{1}+m \cdot \sigma_{1}}{t_{1}}$, where $\rho_{1}$ is the average 1-norm value of all the nonzero rows in $X, \sigma_{1}$ is the standard deviation of all the noise components, $t_{1}$ is a parameter selected from $[2.5,3.5]$. In practice, a conservative determination of the unknown $\sigma_{1}$ can be obtained from the statistical observation over a time period.

Considering that the modification mainly stems from the threshold-based refinement, we refer to the above algorithm as TMOMMP, which is presented in Algorithm 1.

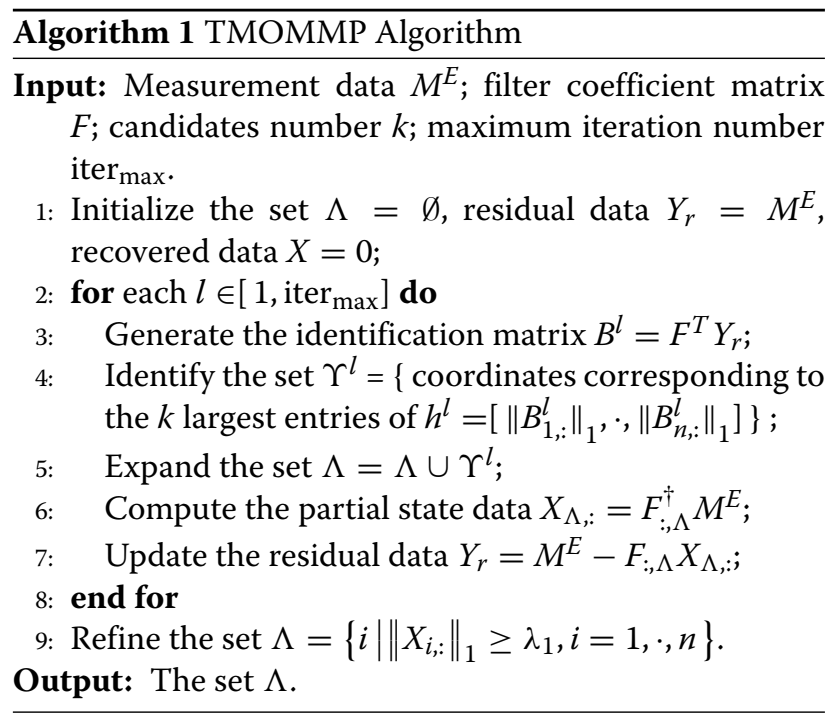

\section{MOMMP based on Gaussian distribution (MOMMP-GD) algorithm}

In addition to the above multi-candidate identification in the former subsection, the direction of identifying an uncertain number of coordinates is still worth studying
[19]. Here, we propose to take full advantages of energy distribution of row vectors of identification matrix.

In each iteration, we firstly determine the available set $\Omega-\Lambda$ and generate the vector $h^{l}=\left[\left\|B_{l_{1},}^{l},\right\|_{1}, \ldots,\left\|B_{l_{d},}^{l},\right\|_{1}\right]$, where $\Omega=\{1, \cdot, n\}, d=|\Omega-\Lambda|$ and $l$ denotes iteration counter. According to the experimental results in [14] and the property $\left\|B_{l_{i},:}^{l},\right\|_{1} \leq \sqrt{m} \cdot\left\|B_{l_{i},}^{l},\right\|_{2}$, we conclude that the elements of $h^{l}$ will also approximately follow the Gaussian distribution. This means only several entries of $h^{l}$ are large and the others are concentrated on some certain positive constant. Thus, we propose to iteratively identify the coordinates, whose corresponding values in $h^{l}$ are near the peak. By $\rho_{2}$ and $\sigma_{2}$, we respectively denote the mean and standard deviation of $h^{l}$. Selecting a parameter $t_{2}$ from $[2.5,3.5]$, we fix the threshold $\lambda_{2}=\rho_{2}+t_{2} * \sigma_{2}$. Secondly, we identify the set $\Upsilon^{l}=\left\{l_{i} \mid h_{i}^{l}>\lambda_{2}, i=1, \cdot, d\right\}$. Through checking whether there is no newly identified coordinate, the detection makes a judgment of termination. If not, the set is expanded as $\Lambda=\Lambda \cup \Upsilon^{l}$. Finally, the partial state data is computed as $X_{\Lambda,:}=F_{:, \Lambda}^{\dagger} M^{E}$ and residual data is updated as $Y_{r}=M^{E}-F_{:, \Lambda} X_{\Lambda,:}$.

Due to the Gaussian distribution of elements of $h^{l}$, we referred to the above algorithm as MOMMP-GD algorithm and show it in Algorithm 2. Compared with the similar AOMP algorithm in [14], we propose to calculate the 1 -norm of each row vector and compute $X_{\Lambda, \text { : }}$ rather than $X_{\Upsilon^{l},:}$. The above modifications significantly improve the probability of exact detection, which can be seen from the extensive numerical experiments. Meanwhile, the MOMMP-GD algorithm adaptively terminates the detection without a prior knowledge of $s_{\max }$ and the standard deviation of all the noise components, which is more promising in CRNs with fewer statistical observations.

\section{Deterministic matrix from MCSGD algorithm}

To improve the detection accuracy, based on the expression in (8), we construct a deterministic matrix by reducing the value of $\left|F_{:, i}^{T} F_{: j}\right|, \forall i \neq j$ while preserving that of $\left|F_{:, i}^{T} F_{:, i}\right|, \forall i \in \Omega$ to be 1 . On the other hand, the system model in the second section indicates that the components of $F$ is required to guarantee an exact recovery of $X$ and should be transmitted to the FC, which practically increases the energy consuming a lot. Fortunately, the above issue can be alleviated by utilizing a deterministic matrix $F$ since it can be constructed and stored in FC beforehand and its transmission becomes unnecessary.

Considering the existence of Welch bound, the direction of optimizing random matrix $F$ as close as possible to an equiangular tight frame (ETF) is more practical, since ETF has the minimal coherence among all matrices of the same dimension. However, the coherence $\mu$, which is defined as the largest absolute and normalized inner product between different columns, is analyzed to 


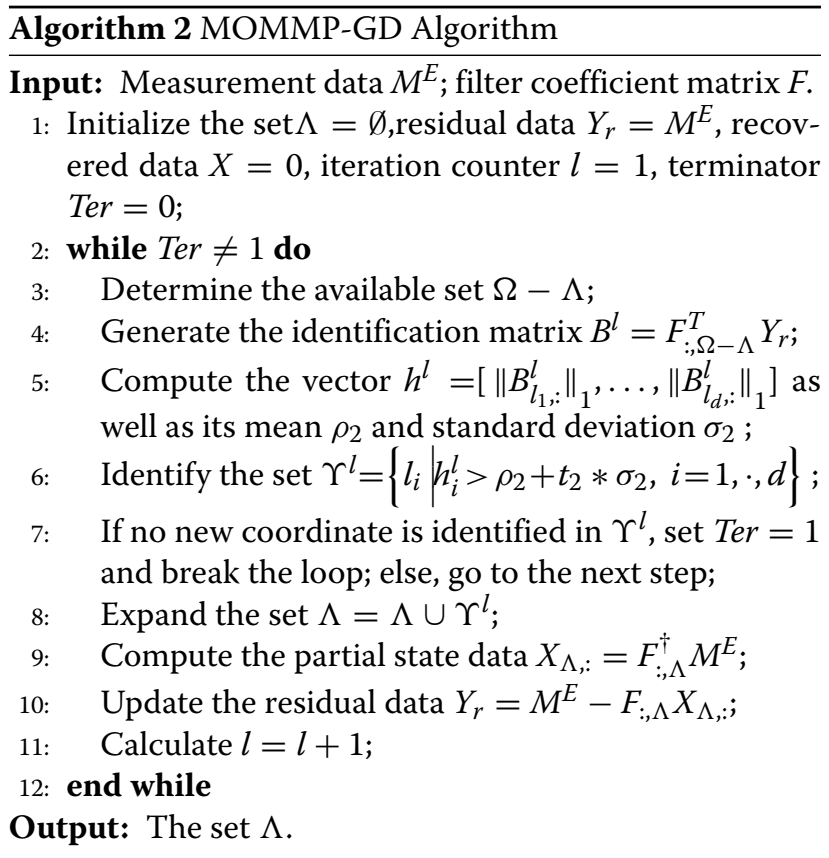

be a pessimistic measure in CS [21]. Thus, we also adjust our main objective to the reduction of the following $t$ averaged coherence [21], which is defined as the average of all absolute and normalized inner products between different columns in $F$ (that is, the off-diagonal elements in Gram matrix $G=F^{T} F$ ) that are above the predefined threshold $t$.

$$
\mu_{t}(F)=\frac{\sum_{1 \leq i, j \leq n \text { and } i \neq j}\left(\left|G_{i, j}\right| \geq t\right)\left|G_{i, j}\right|}{\sum_{1 \leq i, j \leq n \text { and } i \neq j}\left(\left|G_{i, j}\right| \geq t\right)}
$$

For $t=0$, we obtain the average of all the absolute offdiagonal entries of $G$. As $t$ grows, the value of $\mu_{t}(F)$ grows and approaches the coherence $\mu$ from below.

\section{The description of MCSGD algorithm}

In this subsection, we briefly describe how the proposed MCSGD algorithm minimizes $\mu_{t}(F)$. Our approach is inspired by designing optimized projections by using shrinkage process in [21] and gradient descent process in [22]. Consider the following convex set $\mathcal{H}$ which contains the ideal ETFs, the deterministic matrix construction problem can be solved by projecting onto $\mathcal{H}$ alternatively

$$
\mathcal{H}=\left\{H \in R^{n \times n}, H_{i, i}=1,\left|H_{i, j}\right| \leq \mu_{w}, \text { for } i \neq j\right\}
$$

where the Welch bound $\mu_{w}$ is described in (9).

Initializing $F$ as a Gaussian random matrix with normalized columns, we firstly find $H \in \mathcal{H}$ that minimizes $\left\|F^{T} F-H\right\|_{F}^{2}$, and then update $F$ that minimizes $\left\|F^{T} F-H\right\|_{F}^{2}$. The $t$-averaged coherence of $F$ will be gradually reduced. The iterations are carried on until the difference of $\mu_{t}(F)$ between two consecutive iterations is under a tolerable precision or the algorithm has reached a maximum iteration number. After the final iteration, we obtain the optimized and deterministic matrix $F$. Now, let us briefly present the proposed MCSGD algorithm in Algorithm 3.

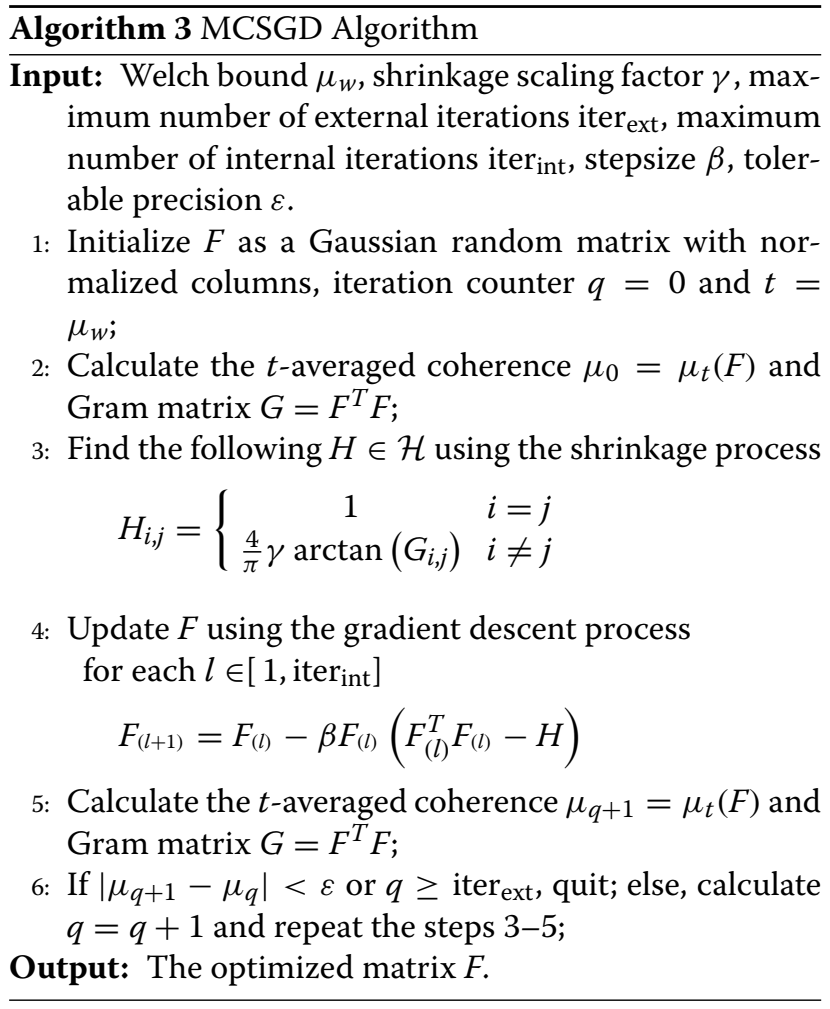

\section{Find $H$ using shrinkage process}

The problem of finding $H \in \mathcal{H}$ is written as

$$
\min _{H \in \mathcal{H}}\|G-H\|_{F}^{2}=\min _{H \in \mathcal{H}}\left(\sum_{i j}\left|G_{i, j}-H_{i, j}\right|^{2}\right) \text { s.t. } G=F^{T} F
$$

According to the definition of Frobenius matrix norm, the minimum can be obtained for matrix $H$ with shrinkage operation to Gram matrix $G$ [21]. In this part, we introduce another simple but effective shrinkage function to obtain the minimum $H$.

$$
H_{i, j}= \begin{cases}1 & i=j \\ \frac{4}{\pi} \gamma \arctan \left(G_{i, j}\right) & i \neq j\end{cases}
$$

where $\gamma \in\left[\mu_{w}, 1\right)$ is the shrinkage factor, which enables to adjust the shrinkage range of the elements in $G$.

The proposed shrinkage function, the shrinkage function in [21], the component-wise approach in [22] and the non-shrinkage function are compared, and their results 
are described graphically in Fig. 1. Take $\gamma=0.4$ as an example, and the variable range of the input valuables is $[-1,1]$. The variable range of the output value with non-shrinkage function remains the same, while that with the other three techniques are $[-0.4,0.4]$. The above results demonstrate that the shrinkage function reduces the variable range of values from $[-1,1]$ to $[-0.4,0.4]$, i.e., the relatively-large absolute off-diagonal entries in $G$ are effectively reduced.

Gaussian random matrix $F \in R^{400 \times 500}$ is adopted to validate the proposed MCSGD algorithm. The input parameters are fixed as: $\beta=0.01$, iter ext $=200$, iter $_{\text {int }}=100$ and $\varepsilon=10^{-4}$. Selecting shrinkage factor $\gamma$ from $\{0.4,0.55,0.65,0.75\}$, we present the corresponding $t$-averaged coherence $\mu_{t}(F)$ in Fig. 2. Obviously, the value of $\mu_{t}(F)$ decreases with iterations. Moreover, a smaller $\gamma$ requires fewer iterations to provide a lower $\mu_{t}(F)$. These simulations experimentally demonstrate the effectiveness of shrinkage process in MCSGD algorithm.

\section{Update $F$ using gradient descent process}

After the implementation of the above shrinkage process, we get a temporal $H \in \mathcal{H}$. The next issue to address is how to update $F$. In each iteration of the algorithm in [21], singular value decomposition (SVD) method is applied to reduce the rank of $H$. Afterwards, its square-root is built and the minimum $F$ is found. However, the above algorithm will create some large values that are not present in the original Gram matrix. Large off diagonal values in $G$ ruin completely the original signal recovery algorithms some times.
In face of the above shortcoming and inspired by [22], we alternatively apply a gradient descent method to solve the second minimization problem and update $F$. Denote the cost function $J=\left\|F^{T} F-H\right\|_{F}^{2}$, the minimization problem is solved by the gradient descent process $F_{i, j} \leftarrow$ $F_{i, j}-\eta \frac{\partial J}{\partial F_{i, j}}$, where $\eta>0$ is the stepsize. According to matrix analysis [36], the following result holds

$$
\begin{aligned}
\frac{\partial J}{\partial F} & =\frac{\partial}{\partial F} \operatorname{Tr}\left\{\left(F^{T} F-H\right)^{T}\left(F^{T} F-H\right)\right\} \\
& =4 F\left(F^{T} F-H\right)
\end{aligned}
$$

where $\operatorname{Tr}\{\cdot\}$ denotes the trace operation. Thus, the update process is written as

$$
F_{(l+1)}=F_{(l)}-\beta F_{(l)}\left(F_{(l)}^{T} F_{(l)}-H\right)
$$

where $l$ is the iteration counter and stepsize $\beta=4 \eta$.

The effect of stepsize $\beta$ is also experimentally investigated. The input parameters are same as that in the previous subsection except $\gamma=0.4$. For each $\beta$ selected from $\{0.001,0.005,0.01,0.03,0.05\}$, the value of $t$-averaged coherence $\mu_{t}(F)$ is regarded as a function of iterations in Fig. 3. It can be seen that both $\mu_{t}(F)$ and number of required iterations decrease as $\beta$ increases, and they finally become stable when $\beta \geq 0.03$.

\section{MCSGD analysis}

According to the result in [37], we mention that constructing ETFs using alternating minimization methods have a global minimum subject to an appropriate initialization. In detail, there exist at least one accumulation point for

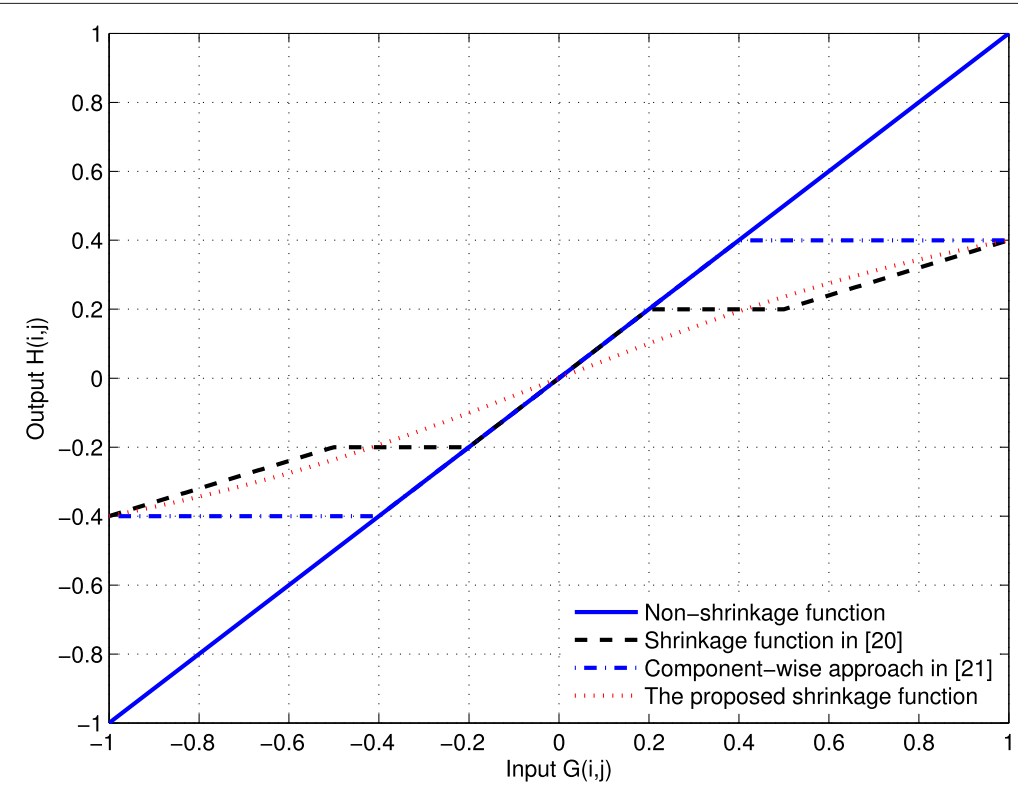

Fig. 1 Applying shrinkage function for $\mu_{w}=0.4$ and $\gamma=0.4$ 


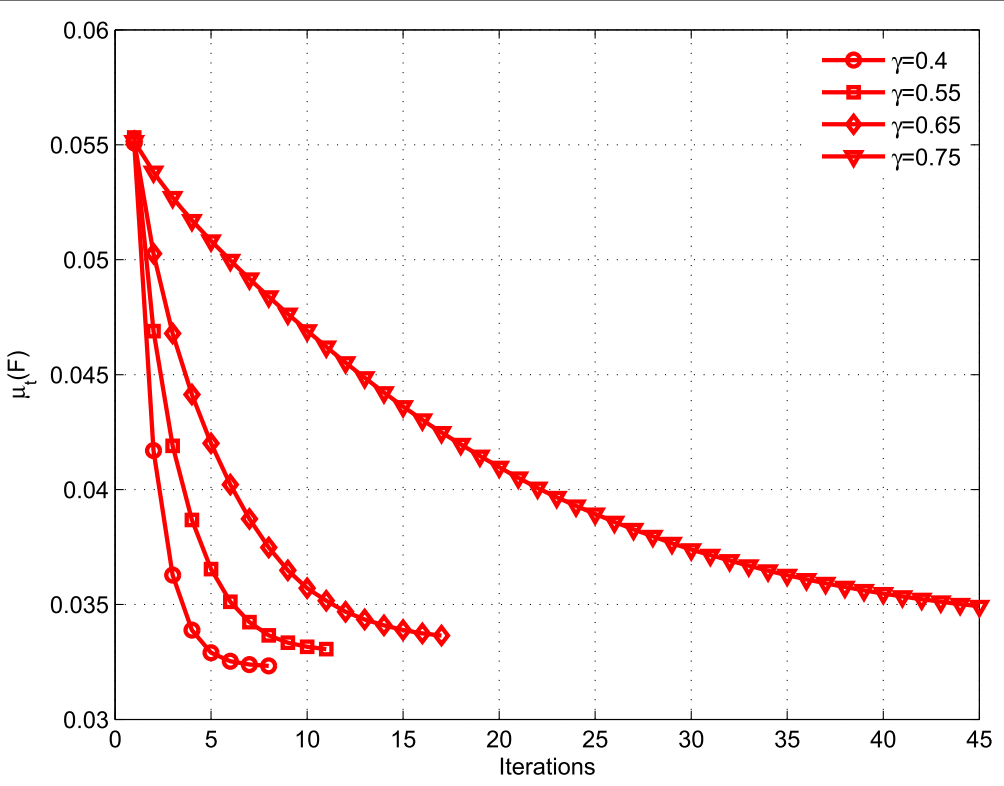

Fig. $2 \mu_{t}(F)$ as a function of iteration and various values of $\gamma$

$\min _{G, H}\|G-H\|_{F}^{2}$, and the minimization leads to a global minimum after infinite number of iterations.

As shown in Algorithm 3, we also solve the matrix construction problem by adopting the alternating projection method from the macro perspective. To be specific, both the shrinkage process and the gradient descend process are used to update $H$ and $F$ by solving two minimization problems in the alternating projection procedure, and the above procedure will be repeated for a number of iterations. Due to the operations in alternating projection, the structure of $G=F^{T} F$ and $H$ changes frequently. Such operations make the set $\mathcal{H}$ cannot maintain the convex sets all the time, which makes the whole problem a nonconvex one. Since the set $\mathcal{H}$ is generally nonconvex, alternating projection may not converge as one might wish. However, it appears that norm convergence always occurs in practice [37]. The convergence of first process is guaranteed only if $\gamma$ is chosen not far from 1 , then

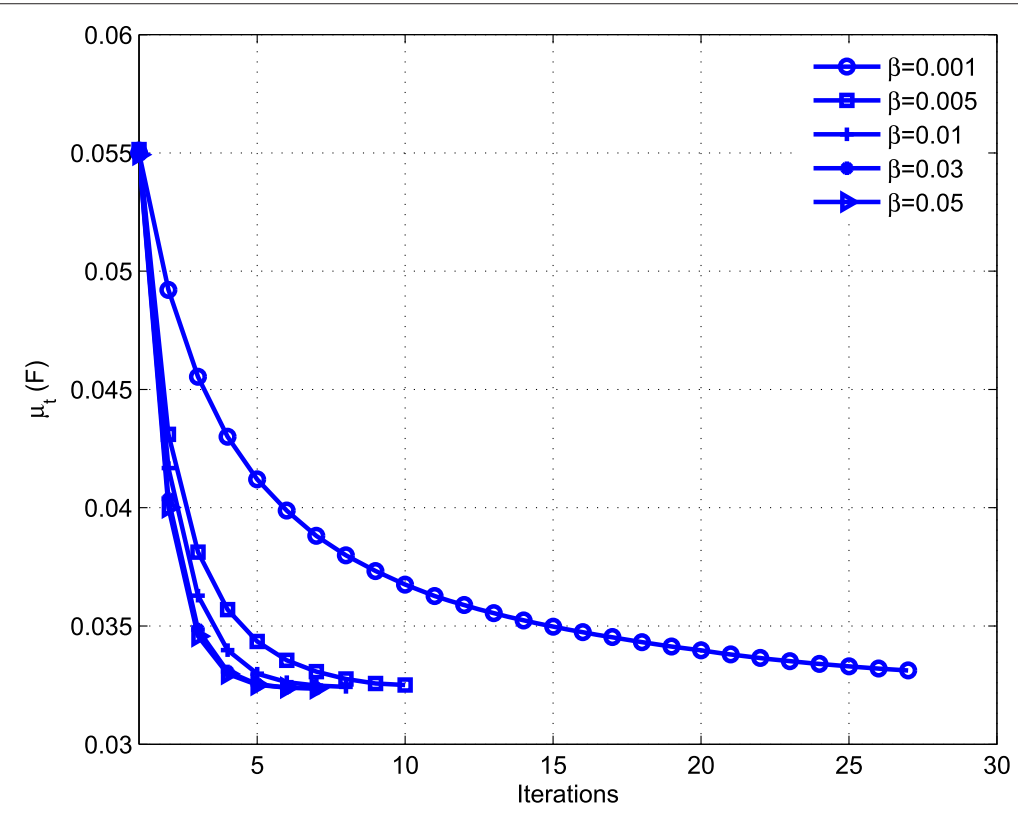

Fig. $3 \mu_{t}(F)$ as a function of iteration and various values of $\beta$ 
the structures of $G$ and $H$ will be changed smoothly and slowly, and their distance becomes shorter in norm measure. Although smaller $\gamma$ leads to much faster convergence rate, $\gamma$ cannot be set too small since it will cause all the offdiagonal entries of Gram matrix breakthrough the Welch bound and even approach to zero, which may lead to badly non-convergence [21]. Facing the above tradeoff, according to the simulation results in the former subsection, we choose $\gamma=0.4$ as a reasonable choice. Referring to second minimization, the proposed method offers a gradient descent method to update the $F$ which guarantees gradual reduction of cost function $J$ and convergence to a local minimum [22].

After the convergence analysis, we perform simulations to experimentally validate the construction performance of MCSGD algorithm. The Gaussian random measurement matrix and deterministic matrices from Elad's algorithm [21] and Gradient algorithm [22] are involved in comparison. For each $p$ varying from 100 to 400 with increment 25 and fixed $n=500$, their values of $t$ averaged coherence $\mu_{t}(F)$ as well as the corresponding Welch bound are computed and shown in Fig. 4. For each $p$, the value of $t$-averaged coherence of $F$ from MCSGD algorithm is much lower than that of other algorithms and closer to the Welch bound. Therefore, the proposed MCSGD algorithm stands out as the best algorithm to provide deterministic matrix $F$.

Combining the above theoretical and experimental results, we conjecture that the deterministic matrix $F$ constructed by MCSGD algorithm further improves the detection performance of MOMMP algorithms. Referring to the realization of deterministic matrix $F$ on each CR user, frequency-selective surfaces (FSSs) [38] and random filtering $[39,40]$ can be utilized as effective approaches.

\section{Numerical experiments}

In this section, we validate the detection accuracy and time of two MOMMP algorithms by performing numerical experiments under different sampling rates, SNRs, numbers of $\mathrm{CR}$ users, and reports, where the deterministic matrix $F$ from MCSGD algorithm is utilized as a filter coefficient matrix.

\section{Simulation setting}

Consider the following CRN: $m$ CR users are uniformly and randomly located within a $500 \times 500 \mathrm{~m}^{2}$ area centered at the FC, and they cooperatively detect the presence of PUs within a $1000 \times 1000 \mathrm{~m}^{2}$ area centered at the same FC, to which $n=500$ channels are licensed. Suppose that only $s$ channels are occupied, and their locations are chosen uniformly at random. A simple model of CRN is illustrated in Fig. 5. Rather than $n$ channel states, only $p$ linear combinations are reported by each CR user. Thus, the measurement data $M \in R^{p \times m}$ instead of state data $X \in R^{n \times m}$ is transmitted from $m$ CR users to $\mathrm{FC}$, where the deterministic matrix $F \in R^{p \times n}$ from MCSGD algorithm is adopted as filter coefficient matrix. During the transmission, $M$ is corrupted by Gaussian noise $N$ with SNR varying from -15 to $30 \mathrm{~dB}$.

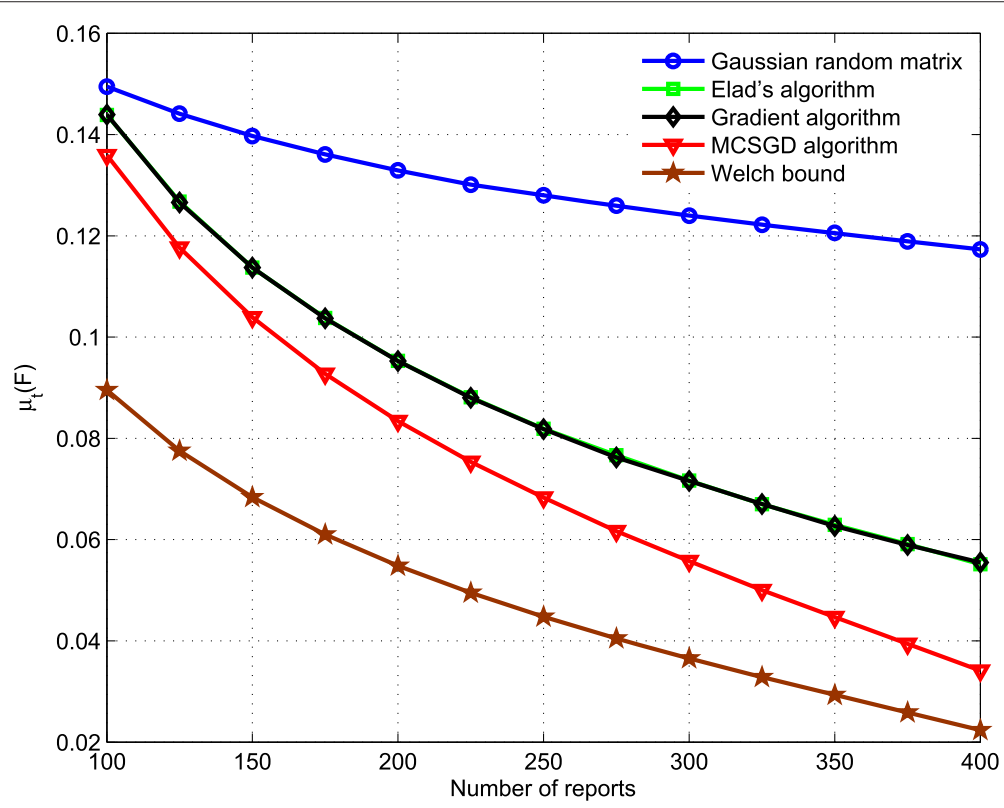

Fig. $4 \mu_{t}(F)$ as a function of $p$ and various algorithms 


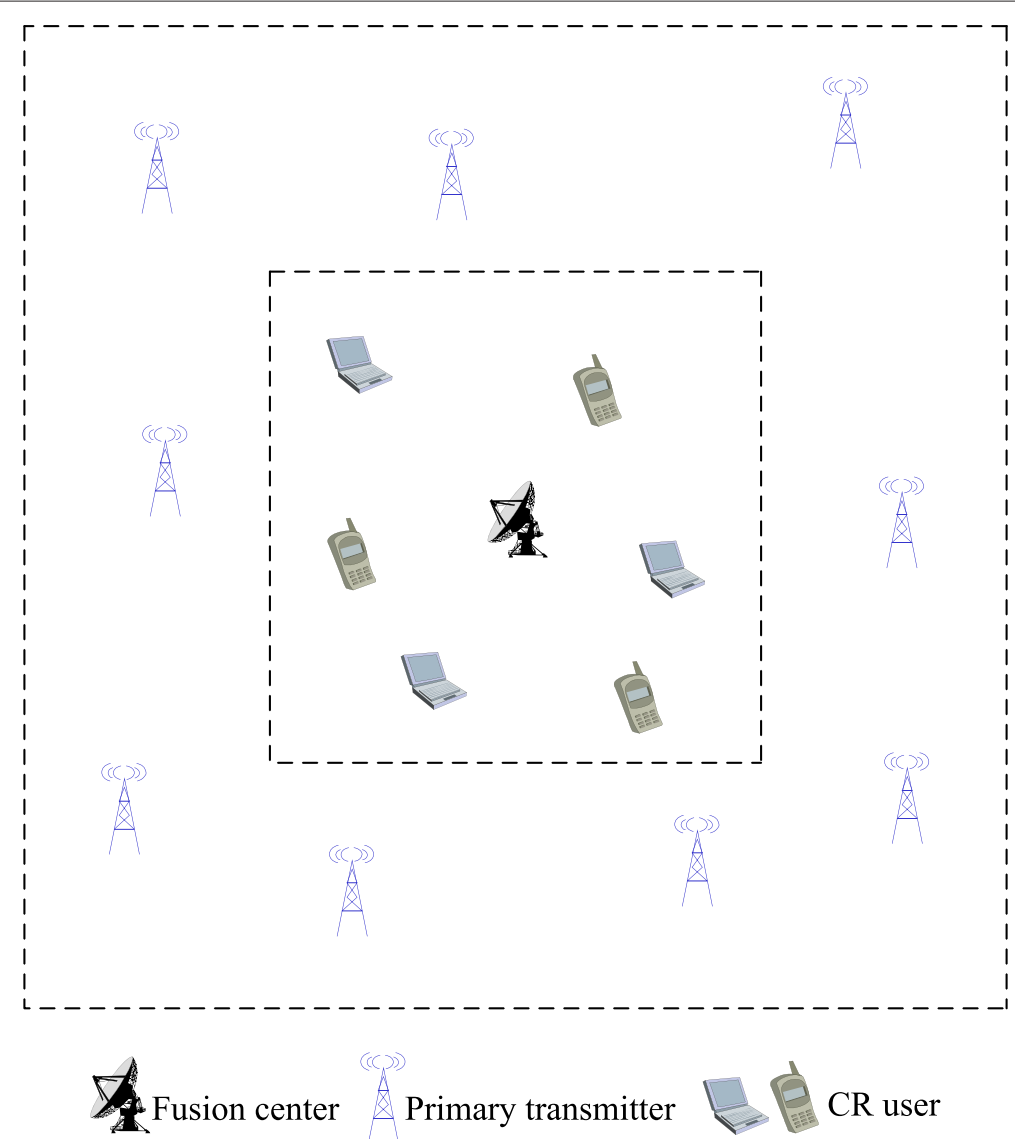

Fig. 5 The model of CRN

To quantify the compression and loss of reports, we respectively define sampling rate and loss rate as follows

$$
\text { sampling rate }=\frac{|E|}{m \times n} ; \text { loss rate }=\frac{|E|}{m \times p}
$$

where $|E|$ means the number of reports received at the FC. After the implementation of MOMMP algorithms, the detection performance is evaluated by computing the following probability of detection $P_{D}$ and probability of false alarm $P_{F}$

$$
P_{D}=\frac{\text { No.Hit }}{\text { No.Hit }+ \text { No.Miss }} ; P_{F}=\frac{\text { No.False }}{\text { No.False }+ \text { No.Miss }}
$$

where No.Hit is the number of exact detections of the presence of PUs, No.Miss is the number of miss detections, No.False is the number of false alarms.

At the beginning, we investigate the influence of candidates number $k$ upon the detection of TMOMMP algorithm. The reason for this is that the corresponding iter $_{\max }=\left\lceil s_{\max } / k\right\rceil$ will greatly affect the detection time. Therefore, a reasonable $k$ requiring less detection time while providing equivalent detection accuracy should be determined. Selecting $k$ from $\{1,5,10,20\}$ and determining its corresponding iter $r_{\max }$ subject to $k *$ iter $_{\max }=s_{\max }=$ 100 , we experimentally obtain all equivalent values of $P_{D}$ and $P_{F}$. For the sake of paper length, we omit the presentation of their experiment results and fix $k=20$ in the following experiments. In addition, to verify the average performance of our proposals in detecting occupied channels, we repeat the experiment with the same scenario $Q=50$ times and show their average values of $P_{D}$ and $P_{F}$ as well as detection times.

\section{Detection accuracy versus sampling rate}

Considering that sampling rate generally demonstrates the transmission loss and report compression, we firstly perform numerical simulations to demonstrate its influence upon the detection performance. Fix the following parameters: number of occupied channel $s=50$, number of reports $p=400$ and SNR $=15 \mathrm{~dB}$. Actually, the case $s=50$ corresponds the spectrum utilization efficiency $10 \%$, which is large enough in practice according to FCC [41] and spectrum utilization efficiency in Singapore [42]. In this part, the AOMP algorithm in [14], jointly sparse recovery with missing observation (JSR-MO) algorithm in 
[17] and centralized reweighed $l_{q}$ (CRLq) algorithms ( $q=$ $1,2)$ in [10] are also involved for comparison. Actually, instead of the time varying $s$ in practice, only $s_{\max }=100$ is available and then utilized in TMOMMP and JSR-MO algorithms.

For each sampling rate varying from 0.1 to 0.8 with increment 0.1 , we calculate its corresponding $P_{D}$ and $P_{F}$ for AWGN fading channel and plot them in Fig. 6. Obviously, from the perspective of $P_{D}$, CRL1 and CRL2 are superior to others in case of serious loss since they can provide higher $P_{D}$ when sampling rate is small. However, when sampling rate exceeds a threshold, namely 0.5 , they are caught up with or even surpassed. On the other hand, CRL1 and CRL2 take thousands of seconds to finish the detection while others require a few seconds, which are shown in next subsection. Generally speaking, among all other greedy-type algorithms, the JSR-MO algorithm seems to be the best except when sampling rate falls between 0.1 and 0.2. Unfortunately, when sampling rate is larger than 0.2 , the higher $P_{F}$ provided by JSR-MO will reduce the throughput [24] since some unoccupied channels are declared as occupied and not allocated to CR users. Compared with AOMP algorithm, the proposed MOMMP-GD algorithm significantly outperforms up to sampling rate $=0.6$. Afterwards, both MOMMP$\mathrm{GD}$ and AOMP algorithms provide perfect detection, i.e., $P_{D} \approx 1$ and $P_{F} \approx 0$. The above superiority experimentally demonstrates the positive effect of 1-norm in measuring the row vector and orthogonal projection in recovering state data. Moreover, the TMOMMP algorithm is superior to AOMP algorithm when sampling rate falls between
0.25 and 0.45 . In terms of $P_{F}$, the proposed TMOMMP and MOMMP-GD algorithms provide almost the same detection as AOMP algorithm. Therefore, these results indicate that the MOMMP-GD algorithm is more suitable for practical application with serious transmission failure.

To validate the robustness of our proposal, we also exploit the Rayleigh fading channels. Keep the above parameters unchanged, we obtain the corresponding $P_{D}$ and $P_{F}$ in Fig. 7. Overall, although CRL1 and CRL2 can provide higher $P_{D}$ when sampling rate $\leq 0.4$, the significantly high $P_{F}$ and computational complexity limit their application in practice. With respect to $P_{D}$, the proposed TMOMMP algorithm is almost the best one in case of serious loss, and JSR-MO algorithm outperforms only when $0.45 \leq$ sampling rate $\leq 0.7$. Moreover, the proposed TMOMMP and MOMMP-GD algorithms always outperform AOMP algorithm except when $0.5 \leq$ sampling rate $\leq 0.7$ the AOMP algorithm is slightly superior to TMOMMP algorithm. From the perspective of $P_{F}$, the MOMMP-GD algorithm is equivalent to AOMP algorithm while the TMOMMP algorithm degrades when $0.2 \leq$ sampling rate $\leq 0.5$, which are much better than CRL1, CRL2, and JSR-MO algorithms. Comparing the results in Figs. 7 and 6, we conclude that better detection performance is obtained when Rayleigh fading channel is utilized as a sensing channel. For instance, to obtain $P_{D} \geq$ 0.9 from all three algorithms, sampling rate $=0.47$ is enough for Rayleigh fading channel while sampling rate = 0.52 for AWGN fading channels. Thus, the above results demonstrate that a proper choice of algorithm greatly benefits the detection in certain fading channel.

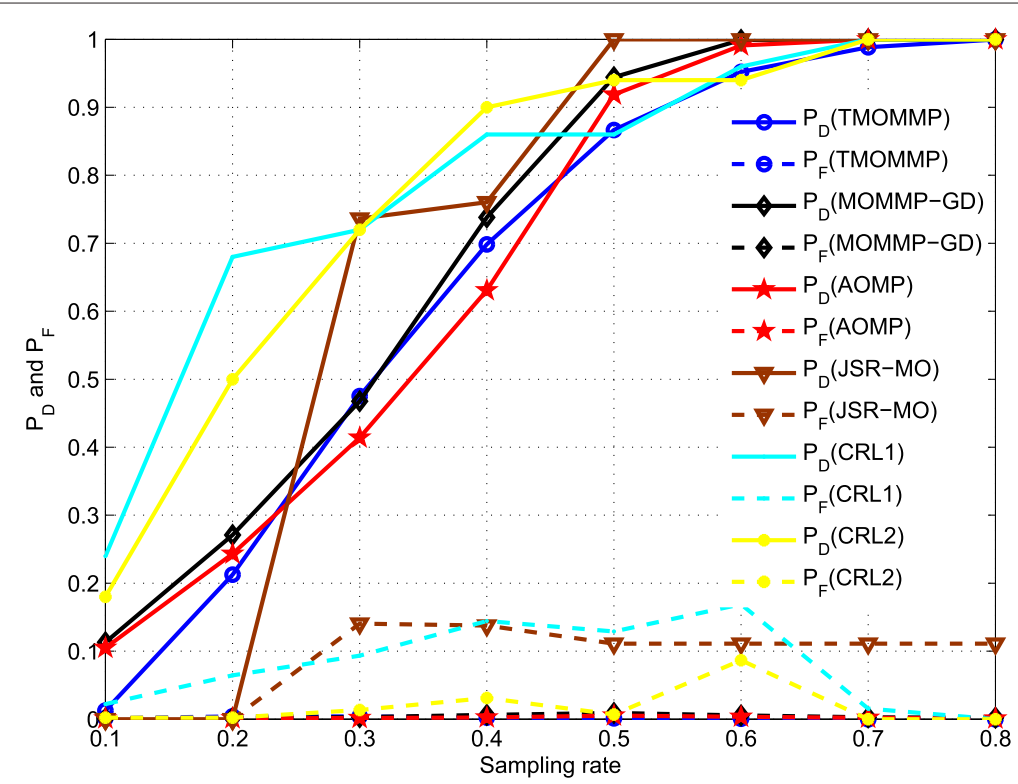

Fig. $6 P_{D}$ and $P_{F}$ as functions of sampling rate for AWGN fading channel 


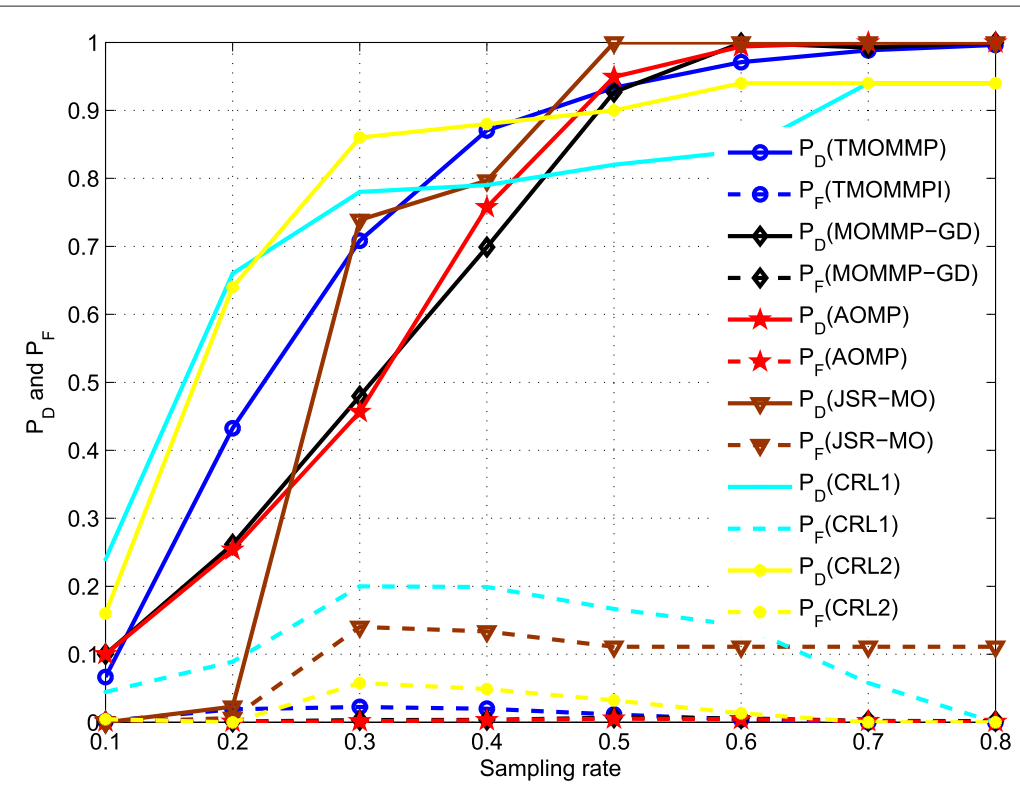

Fig. $7 P_{D}$ and $P_{F}$ as functions of sampling rate for Rayleigh fading channel

Combining the above results, we conclude that our proposal provide higher probability of exactly detecting occupied channels.

\section{Detection time versus sampling rate}

In addition to detection accuracy, computational complexity is also an important evaluation indicator. The reason is that, in order not to interfere the PUs, CR users should detect the their presence with a high probability and vacate the channels or reduce transmit power within a certain amount of time. In other words, the time window for detection should be small enough. In each window with fixed duration, the first part is used for performing spectrum sensing and the rest is reserved for transmitting data. During the sensing interval, all CR users keep quiet as enforced by protocols, e.g., at the media access control layer. Therefore, an efficient detection algorithm can benefit the improvement of throughput.

In this part, we choose detection time to demonstrate the detection efficiency. During the simulations in Fig. 6, we record the consumed time for each trial and then calculate the average detection time for each algorithm. Note that, CRL1 and CRL2 are not compared in Fig. 8 since that they involve massive calculation and are time-consuming. It is obvious that, among all the compared algorithms, the proposed MOMMP-GD and AOMP algorithms outperform as they consume almost the same and least time to finish the detection, and the proposed TMOMMP algorithm is slightly worse than them. Unfortunately, the JSR-MO algorithm always takes the most time and is the worst one. Therefore, we validate the superiority of our proposals with respect to computational complexity. Whenever on the same parameter for Rayleigh fading channels, the obtained average detection times are almost the same as that for AWGN fading channel. Therefore, we validate that our proposals are superior with respect to computational complexity, which are of great significance to the real-time CRNs.

\section{Detection accuracy versus SNR}

Considering the measurement data is inevitably perturbed in transmission, we investigate the robustness of our proposals. Fixing $s=50, p=400$, and sampling rate $=0.8$, and varying SNR from -15 to $30 \mathrm{~dB}$ with increment $5 \mathrm{~dB}$, we adopt AWGN fading channel and validate the detection performance of our proposals. From Fig. 9, we can see that the value of $P_{D}$ increases with SNR. At a high noise level, e.g., $\mathrm{SNR}=-5 \mathrm{~dB}$, the TMOMMP algorithm is obviously superior to MOMMP-GD algorithm in the sense of $P_{D}$ while the MOMMP-GD algorithm significantly outperforms with respect to $P_{F}$ due to the non-existence of false alarm. In MOMMP-GD algorithm, high noise level will destruct the property of approximate Gaussian distribution, which results in the identification of fewer coordinates and hence small $P_{D}$ and $P_{F}$. On the contrary, as for TMOMMP algorithm in the same scenario, the range of difference between $h_{i}, \forall i \in \Gamma$ and $h_{i}, \forall i \notin \Gamma$ is reduced. Thus, a larger $\lambda_{1}$ is required to identify suitable coordinates. However, the utilization of $t_{1}$ makes $\lambda_{1}$ smaller, resulting in larger $P_{D}$ and $P_{F}$. Therefore, we propose to choose the detection algorithm by balancing $P_{D}$ and $P_{F}$ in practical application. 


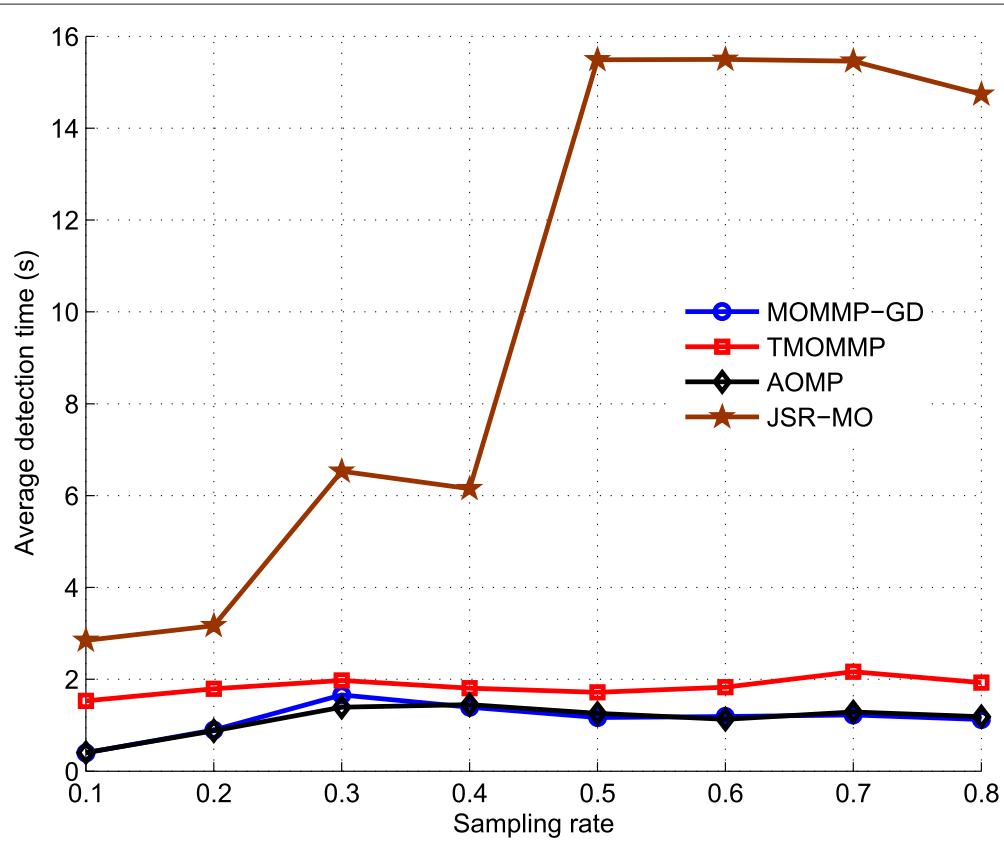

Fig. 8 Average detection time as a function of sampling rate for AWGN fading channel

\section{Detection accuracy versus number of $C R$ users}

Compared with spectrum sensing implemented by single CR user, CSS exploits the spatial diversity from several cooperating $\mathrm{CR}$ users to improve the detection performance. However, its energy consumption overhead can be significant if the number of cooperating $\mathrm{CR}$ users or the number of reports is large. The second issue will be discussed in the next subsection. The algorithm requiring fewer cooperating $C R$ users while providing an equivalent detection performance is more promising to improve the whole energy efficiency. Here, we investigate the influence of number of cooperating CR users $m$ upon the detection performance over AWGN fading channel. Setting $s=50, p=400$, sampling rate $=0.6$, and SNR $=$ $15 \mathrm{~dB}$, and varying $m$ from 1 to 10 , we obtain and present the corresponding $P_{D}$ and $P_{F}$ in Fig. 10. Obviously, the

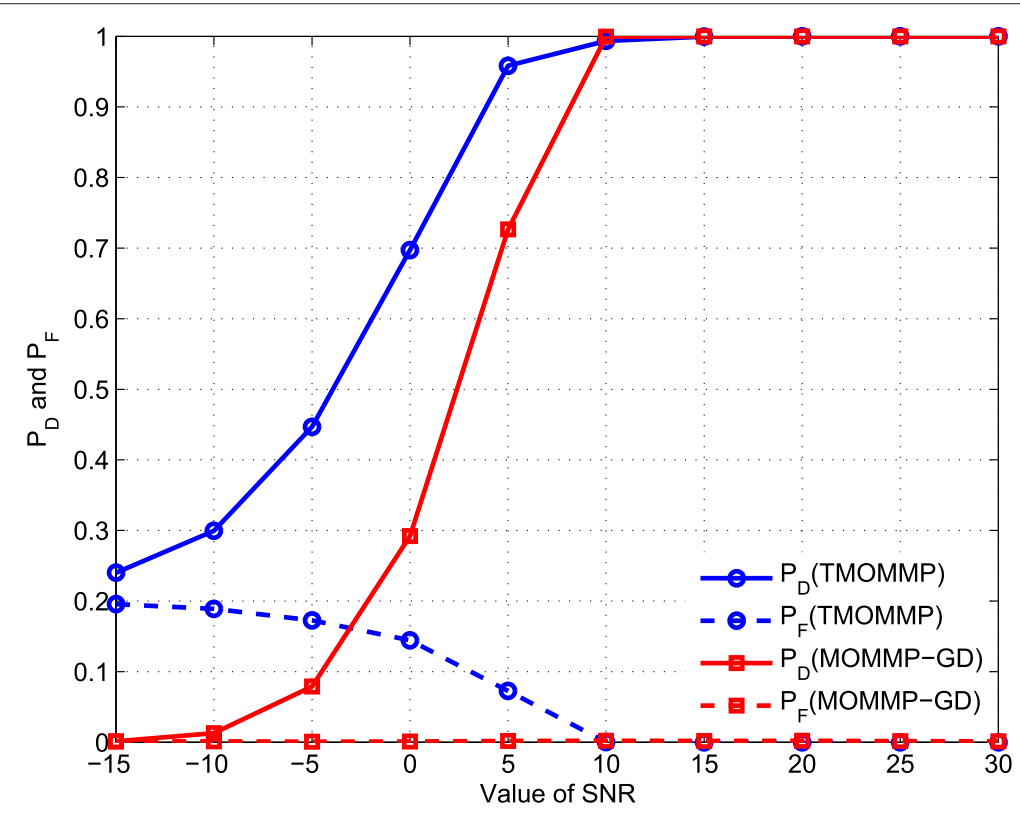

Fig. $9 P_{D}$ and $P_{F}$ as functions of SNR for AWGN fading channel 


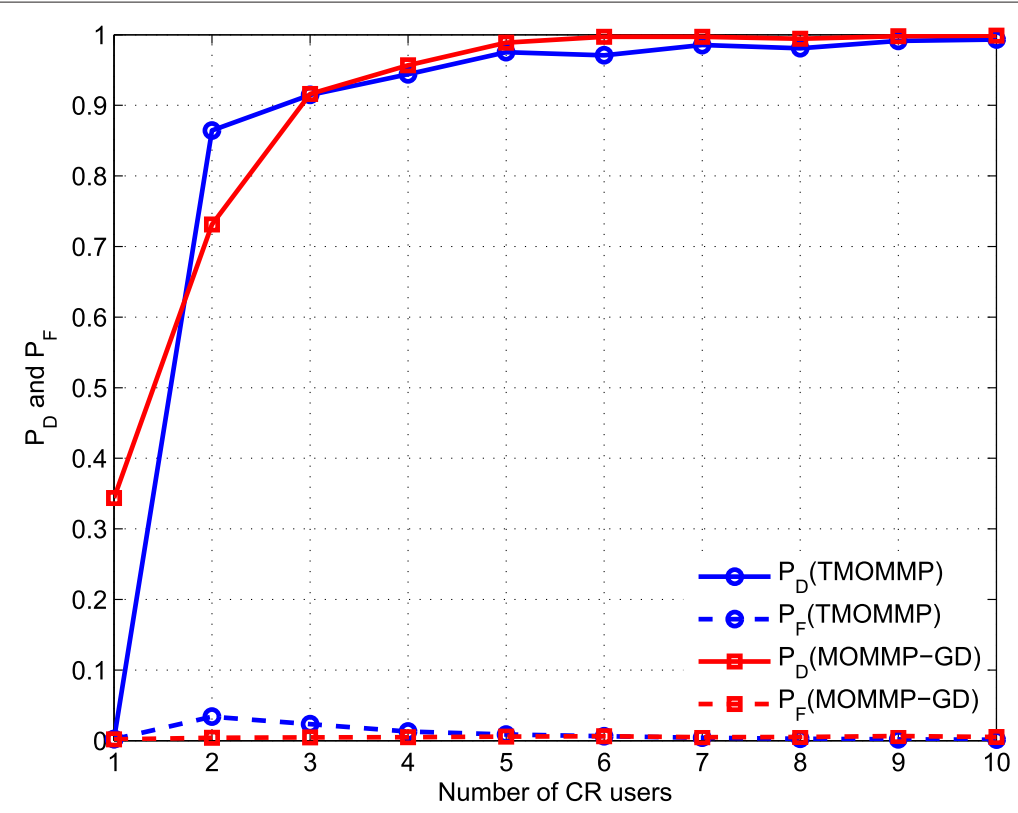

Fig. $10 P_{D}$ and $P_{F}$ as functions of number of $C R$ users for AWGN fading channel

MOMMP-GD algorithm is always superior to TMOMMP algorithm except when $m$ is about 2 . To be specific, with detection accuracy constraint $P_{D} \geq 0.95$, the TMOMMP algorithm requires $m \geq 5 \mathrm{CR}$ users while the MOMMPGD algorithm $m=4 \mathrm{CR}$ users.

\section{Detection accuracy versus number of reports}

Compared with the conventional CSS, CCSS utilizes a deterministic $F \in R^{p \times n}$ to obtain $p$ incoherent linear combinations of $n$ reports on each CR user, achieving report compression. Then, a problem intuitively arises: how many linear combinations are enough to guarantee an exact detection of $s$ occupied channels. Here, we calculate $P_{D}$ and $P_{F}$ for different values of $p$ to address the above problem. The input parameters are fixed as $s=50$, loss rate $=1$, and $\mathrm{SNR}=15 \mathrm{~dB}$. For each $p$ varying from 100 to 400 with increment 25 , we obtain the corresponding $P_{D}$ and $P_{F}$ in Fig. 11 to illustrate the influence of $p$

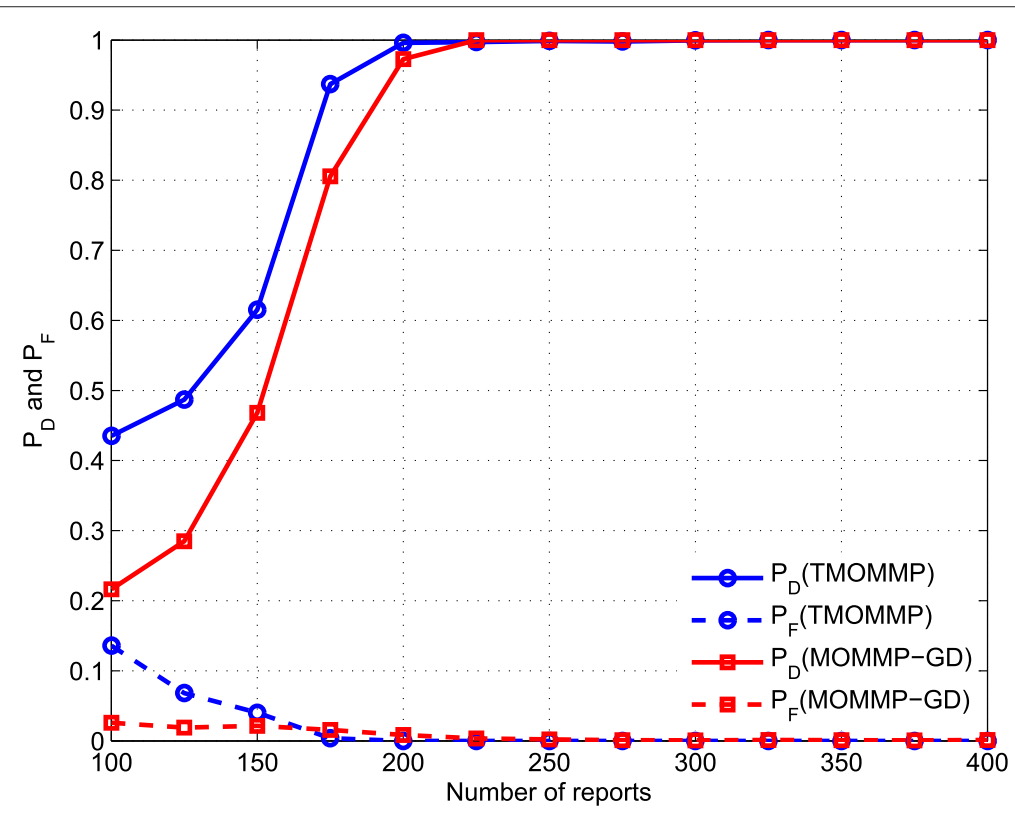

Fig. $11 P_{D}$ and $P_{F}$ as functions of number of reports for AWGN fading channel 
over AWGN fading channel. Obviously, the TMOMMP algorithm always provides higher $P_{D}$ than MOMMP-GD up to $p=225$. However, only when $p \geq 175$, the TMOMMP algorithm is also superior with respect to $P_{F}$. Thus, the TMOMMP algorithm is empirically validated to be a competitive detection algorithm.

\section{Conclusions}

In this paper, we firstly proposed MOMMP algorithms to efficiently detect occupied channels in CRN, in which multi-candidate identification reduces the number of required iterations but does not degrade the detection accuracy. Secondly, two common but different approaches were introduced to realize the multi-candidate identification. With a prior knowledge of $s_{\max }$, the TMOMMP algorithm iteratively identified a fixed number of coordinates till a preset maximum iteration number, followed by a threshold-based refinement. Alternatively, without any prior knowledge, the MOMMP-GD algorithm iteratively identified an uncertain number of coordinates until there was no new coordinate. And then, deterministic matrix of low $t$-average coherence from the proposed MCSGD algorithm further improved the efficiency of CRN since the number of reports was reduced with detection performance constraint and the transmission of deterministic filter coefficient matrix was unnecessary. Finally, numerical simulations under different influencing factors were performed to validate that our proposals provide satisfactory performance with respect to $P_{D}, P_{F}$ and detection time. Specifically, our proposals are more suitable for practical application with serious transmission failure and noise perturbation. In the further, we will investigate the application of the proposed MOMMP algorithms in other jointly sparse recovery problems.

\section{Competing interests}

The authors declare that they have no competing interests.

\section{Acknowledgements}

This work was financially supported by National Science Foundations of China (No.61174016).

\section{Author details}

${ }^{1}$ Department of Control Science and Engineering, Harbin Institute of Technology, 150001 Harbin, China. ${ }^{2}$ School of Computer Science and Engineering, Hebei University of Technology, 300401 Tianjin, China.

Received: 9 February 2015 Accepted: 29 August 2015

Published online: 22 September 2015

\section{References}

1. J Mitola, GQ Maguire Jr, Cognitive radio: making software radios more personal. Pers. Commun. IEEE. 6(4), 13-18 (1999)

2. $Y$ Zeng, $Y$ - C Liang, AT Hoang, R Zhang, A review on spectrum sensing for cognitive radio: challenges and solutions. EURASIP J. Adv. Sign. Process. 2010, 15 (2010)

3. A Ghasemi, ES Sousa, in New Frontiers in Dynamic Spectrum Access Networks (DySPAN), 2005 First IEEE International Symposium on. Collaborative spectrum sensing for opportunistic access in fading environments, (2005), pp. 131-136
4. J Lundén, V Koivunen, A Huttunen, HV Poor, Collaborative cyclostationary spectrum sensing for cognitive radio systems. IEEE Trans. Signal Process. 57(11), 4182-4195 (2009)

5. L Zhang, Q Wu, G Ding, S Feng, J Wang, Performance analysis of probabilistic soft SSDF attack in cooperative spectrum sensing. EURASIP J. Adv. Signal Process. 2014(1), 1-9 (2014)

6. J Wang, S Feng, Q Wu, X Zheng, Y Xu, G Ding, A robust cooperative spectrum sensing scheme based on Dempster-Shafer theory and trustworthiness degree calculation in cognitive radio networks. EURASIP J. Adv. Signal Process. 2014(1), 1-12 (2014)

7. DL Donoho, Compressed sensing. IEEE Trans. Inf. Theory. 52(4), 1289-1306 (2006)

8. J Meng, W Yin, H Li, E Hossain, Z Han, Collaborative spectrum sensing from sparse observations in cognitive radio networks. IEEE J. Selected Areas Commun. 29(2), 327-337 (2011)

9. F Zeng, C Li, Z Tian, Distributed compressive spectrum sensing in cooperative multihop cognitive networks. IEEE J. Selected Topics Signal Process. 5(1), 37-48 (2011)

10. Q Ling, Z Wen, W Yin, Decentralized jointly sparse optimization by reweighted minimization. IEEE Trans. Signal Process. 61(5), 1165-1170 (2013)

11. M Mishali, YC Eldar, From theory to practice: Sub-nyquist sampling of sparse wideband analog signals. IEEE J. Sel. Top. Signal Process. 4(2), 375-391 (2010)

12. SG Mallat, Z Zhang, Matching pursuits with time-frequency dictionaries. IEEE Trans. Signal Process. 41(12), 3397-3415 (1993)

13. L Jiao, J Chen, J Wu, X Wang, S Zhang, Efficient collaborative spectrum sensing with low sample rate. Wirel. Pers. Commun. 67(4), 923-936 (2012)

14. C Jianrui, L Jiao, J Wu, X Wang, Compressive spectrum sensing in the cognitive radio networks by exploiting the sparsity of active radios. Wirel. Netw. 19, 661-671 (2013)

15. JA Tropp, AC Gilbert, Signal recovery from random measurements via orthogonal matching pursuit. IEEE Trans. Inf. Theory. 53(12), 4655-4666 (2007)

16. W Dai, O Milenkovic, Subspace pursuit for compressive sensing signal reconstruction. IEEE Trans. Inf. Theory. 55(5), 2230-2249 (2009)

17. S Jin, X Zhang, in Global Communications Conference (GLOBECOM), 2014 IEEE. Collaborative compressive spectrum sensing with missing observations for Cognitive Radio networks, (2014), pp. 828-833

18. J Wang, S Kwon, B Shim, Generalized orthogonal matching pursuit. IEEE Trans. Signal Process. 60(12), 6202-6216 (2012)

19. DL Donoho, Y Tsaig, I Drori, J-L Starck, Sparse solution of underdetermined systems of linear equations by stagewise orthogonal matching pursuit. IEEE Trans. Inf. Theory. 58(2), 1094-1121 (2012)

20. EJ Candes, The restricted isometry property and its implications for compressed sensing. C. R. Math. 346(9), 589-592 (2008)

21. M Elad, Optimized projections for compressed sensing. IEEE Trans. Signal Process. 55(12), 5695-5702 (2007)

22. V Abolghasemi, S Ferdowsi, S Sanei, A gradient-based alternating minimization approach for optimization of the measurement matrix in compressive sensing. Signal Proc. 92(4), 999-1009 (2012)

23. Y Wang, Z Tian, C Feng, Sparsity order estimation and its application in compressive spectrum sensing for cognitive radios. IEEE Trans. Wireless Commun. 11(6), 2116-2125 (2012)

24. H Sun, W-Y Chiu, A Nallanathan, Compressive power spectral density estimation. Commun. Lett. IEEE. 16(11), 1812-1815 (2012)

25. MA Lexa, ME Davies, JS Thompson, J Nikolic, in Acoustics, Speech and Signal Processing (ICASSP), 2011 IEEE International Conference on. Compressive power spectral density estimation, (2011), pp. 3884-3887

26. DD Ariananda, G Leus, Compressive wideband power spectrum estimation. IEEE Trans. Signal Process. 60(9), 4775-4789 (2012)

27. C-P Yen, Y Tsai, X Wang, Wideband spectrum sensing based on sub-nyquist sampling. IEEE Trans. Signal Process. 61(12), 3028-3040 (2013)

28. D Dony Ariananda, D Romero, G Leus, in Sensor Array and Multichannel Signal Processing Workshop (SAM), 2014 IEEE 8th. Cooperative compressive power spectrum estimation, (2014), pp. 97-100

29. P Pal, PP Vaidyanathan, in Digital Signal Processing Workshop and IEEE Signal Processing Education Workshop (DSP/SPE), 2011 IEEE. Coprime sampling and the MUSIC algorithm, (2011), pp. 289-294 
30. D Cohen, YC Eldar, Sub-nyquist sampling for power spectrum sensing in cognitive radios: A unified approach. IEEE Trans. Signal Process. 62(15), 3897-3910 (2014)

31. O Mehanna, N Sidiropoulos, Frugal sensing: Wideband power spectrum sensing from few bits. IEEE Trans. Signal Process. 61(10), 2693-2703 (2013)

32. G Leus, DD Ariananda, Power spectrum blind sampling. IEEE Signal Process. Lett. 18(8), 443-446 (2011)

33. D Romero, G Leus, Wideband spectrum sensing from compressed measurements using spectral prior information. IEEE Trans. Signal Process. 61(24), 6232-6246 (2013)

34. Wireless communications: principles and practice, 2nd Edition. (Prentice Hall, New Jersey, NJ, USA, 2012)

35. JA Tropp, Greed is good: Algorithmic results for sparse approximation. IEEE Trans. Inf. Theory. 50(10), 2231-2242 (2004)

36. Matrix analysis, 2nd Edition. (Cambridge university press, New York, NY, USA, 2012)

37. J Tropp, IS Dhillon, RW Heath Jr, T Strohmer, et al, Designing structured tight frames via an alternating projection method. IEEE Trans. Inf. Theory. 51(1), 188-209 (2005)

38. R Mittra, CH Chan, T Cwik, Techniques for analyzing frequency selective surfaces-a review. Proceedings of the IEEE. 76(12), 1593-1615 (1988)

39. JA Tropp, MB Wakin, MF Duarte, D Baron, RG Baraniuk, in Acoustics, Speech and Signal Processing, 2006. ICASSP 2006 Proceedings. 2006 IEEE International Conference on. Random filters for compressive sampling and reconstruction, vol. 3, (2006), pp. III 872-1II 875

40. J Zhang, Y Ban, D Zhu, G Zhang, Random filtering structure-based compressive sensing radar. EURASIP J. Adv. Signal Process. 2014(1), 1-11 (2014)

41. Federal Communications Commission and others, Spectrum policy task force report. ET Docket. 2(135), (2002)

42. MH Islam, CL Koh, SW Oh, X Qing, YY Lai, C Wang, et al, in Cognitive Radio Oriented Wireless Networks and Communications, 2008. CrownCom 2008. $3 r d$ International Conference on. Spectrum survey in Singapore: Occupancy measurements and analyses, (2008), pp. 1-7

\section{Submit your manuscript to a SpringerOpen ${ }^{\circ}$ journal and benefit from:}

- Convenient online submission

- Rigorous peer review

- Immediate publication on acceptance

- Open access: articles freely available online

- High visibility within the field

- Retaining the copyright to your article

Submit your next manuscript at $\boldsymbol{~ s p r i n g e r o p e n . c o m ~}$ 